\title{
Search for QCD Instantons using the H1 experiment at HERA
}

\section{Paweł SOPICKI*广}

IFJ PAN, Cracow (Poland)

E-mail: sopicp@mail.desy.de

Signals of QCD instanton-induced processes are searched for in deep-inelastic scattering (DIS) at the electron-proton collider HERA in the kinematic region defined by the Bjorken-scaling variable $x>10^{-3}$, the inelasticity $0.2<y<0.7$ and the photon virtuality $150<Q^{2}<15000 \mathrm{GeV}^{2}$. The search is performed using $\mathrm{H} 1$ data corresponding to an integrated luminosity of $357 \mathrm{pb}^{-1}$. The instanton-induced scattering processes are modeled by the Monte Carlo generator QCDINS. No evidence for the production of QCD instanton-induced events is observed and upper limit of 1.6 $\mathrm{pb}$ is set on the instanton cross-section at $95 \%$ confidence level, thus excluding the prediction of $10 \pm 2 \mathrm{pb}$.

XXII. International Workshop on Deep-Inelastic Scattering and Related Subjects,

28 April - 2 May 2014

Warsaw, Poland

\footnotetext{
*Speaker.

$\dagger$ On behalf of the H1 Collaboration.
} 


\section{Introduction}

The Standard Model of particle physics contains anomalous processes which violate the conservation of baryon and lepton number $(B+L)$ in the case of electroweak interactions and chirality in the case of strong interactions [1]. Such anomalous processes are induced by instantons 迎, 国. In quantum chromodynamics (QCD), the theory of the strong interactions, instantons are non-perturbative fluctuations of the gluon field. They can be interpreted as tunnelling transitions between topologically non-equivalent vacua.

Up to date, there is no direct experimental evidence for this basic non-perturbative QCD effect.

\section{Phenomenology of QCD Instanton-Induced Processes in DIS}

According to Ringwald and Schrempp [3, 伅, instanton induced reactions in deep-inelastic scattering (DIS) dominantly occur in a photon gluon $(\gamma g)$ fusion process (Fig. 1).

A photon splits into a quark anti-quark pair $\left(q^{\prime}+q^{\prime \prime}\right)$ in the presence of an instanton or an antiinstanton field. The instanton induced reaction $q^{\prime}+g \stackrel{(I, \bar{I})}{\rightarrow} X$ produces five light-flavoured quarks or antiquarks and in addition a number of gluons, $\left\langle n_{g}\right\rangle \sim \mathscr{O}\left(1 / \alpha_{s}\right) \sim 3$ on average. The quarks and gluons emerging from the instanton subprocess are isotropically distributed in the instanton rest system defined by $\overrightarrow{q^{\prime}}+\vec{g}=0$. One expects to find the decay particles in a pseudo-rapidity $(\eta)$ region with a width of typically 2 units. This region is densely populated with particles of relatively high transverse momentum which are homogeneously distributed in azimuth in the instanton rest frame. Apart from this pseudo-rapidity band, the hadronic final state also contains a current jet emerging from the outgoing current quark $q^{\prime \prime}$. The large number of partons emitted in the instanton process leads to a high multiplicity of charged and neutral particles in every event.

For $Q^{\prime 2}>113 \mathrm{GeV}^{2}, x^{\prime}>0.35$ [3] and the kinematic domain in which this analysis is performed, i.e. $0.2<y<0.7, x>10^{-3}$ and $150<Q^{2}<15000 \mathrm{GeV}^{2}$, the predicted crosssection is $\sigma_{\text {HERA }}^{(I)}=10_{-2}^{+2} \mathrm{pb}$. The uncertainty follows from the uncertainty of the strong coupling $\alpha_{s}\left(M_{Z}\right)=0.1184 \pm 0.0007$.

\section{Experimental Method}

Events containing a scattered electron are selected in the phase space region defined by $0.2<$ $y<0.7, x>10^{-3}$ and $150<Q^{2}<15000 \mathrm{GeV}^{2}$. Observables used to discriminate the instanton induced contribution from the standard DIS processes are based on the hadronic final state (HFS). All HFS objects are boosted to the hadronic centre-of-mass frame (HCM). Jets are defined by the inclusive $k_{T}$ algorithm as implemented in FastJet, with the massless $P_{T}$ recombination scheme. The variable $Q^{\prime 2}$ is reconstructed from the particles associated with the current jet (most energetic jet) and the photon 4-momentum, which is obtained using the measured momentum of the scattered electron.

Next, HFS components of the current jet are removed and a band in pseudo-rapidity with a width of \pm 1.1 units in $\eta$ (instanton band) is defined. The number of charged particles (tracks) in the instanton band is counted $\left(n_{B}\right)$ and the total scalar transverse energy of all hadronic final state objects in the instanton band is measured $\left(E_{T, B}\right)$. 
The instanton rest frame, where instanton decays isotropically, is reconstructed as $\overrightarrow{q^{\prime}}+\xi \vec{P}=0$ where $\xi=0.076$ is a constant determined from studies of the instanton simulation. In this system the sphericity $\left(\mathrm{Sph}_{\mathrm{B}}\right)$ and few Fox-Wolfram moments $\left(\mathrm{H}_{10}\right)$ are calculated [8, 8]. Furthermore, the axes $\vec{i}_{\text {min }}$ and $\vec{i}_{\text {max }}$ are found for which in the instanton rest system the summed projections of the 3-momenta of all hadronic final state objects in the instanton band are minimal or maximal [凹]. The relative difference between $E_{I N}=\sum_{h}\left|\vec{p}_{h} \cdot \vec{i}_{\text {max }}\right|$ and $E_{\text {OUT }}=\sum_{h}\left|\vec{p}_{h} \cdot \vec{i}_{\text {min }}\right|$ is called $\Delta_{B}=$ $\left(E_{I N}-E_{\text {OUT }}\right) / E_{\text {OUT }}$. For isotropic events $\Delta_{B}$ is small while for pencil-like events $\Delta_{B}$ is large.

The observable $x^{\prime}$ is reconstructed as $x_{\text {rec }}^{\prime}=\left(x_{1}^{\prime}+x_{2}^{\prime}\right) / 2$ where $x_{i}^{\prime}=Q_{\text {rec }}^{\prime 2} /\left(W_{I, i}^{2}+Q_{\text {rec }}^{\prime 2}\right)$ with $W_{I, 1}^{2}=\left(q_{\text {rec }}^{\prime}+\langle\xi\rangle P\right)^{2}$ and $W_{I, 2}^{2}=\left(\sum_{i} v_{i, \text { rec }}\right)_{\text {Band }}^{2}$.

The dominant experimental systematic uncertainties are related to the energy of the scattered electron and the hadronic energy scale of the HFS. For the limits calculation the dominant systematic uncertainties are related to the signal and background models. There is the signal MC (QCDINS []]) normalisation related to the $\alpha_{s}$ error and there is the shape and normalisation of the background taken from DJANGOH [6] or alternatively from RAPGAP [5].

\section{Search for Instanton-Induced Events}

Multivariate discrimination techniques have been employed to optimize the sensitivity to the instanton processes. The PDERS (Probability Density Estimator with Range Search) method as implemented in TMVA ROOT package [10] has been used.

The strategy to reduce the standard DIS background was based on the observables: $E_{T, J e t}, n_{B}$, $x_{r e c}^{\prime}, \Delta_{B}$ and $E_{I N}$ (Fig. 2). These observables have been chosen since they provide the best signal to background separation. Moreover, their distributions are well described by the Monte Carlo simulations and finally, taking into account the systematic uncertainties, the resulting discriminator distribution is described in the background dominated region.

The distribution of the discriminator is shown in Fig. 3. The simulated background events are mainly concentrated at low discriminator values while the simulated instanton signal events are peaked at the large discriminator values.

In the instanton dominated region the DJANGOH Monte Carlo describes the data, while RAPGAP is above. Since no evidence for QCD instanton-induced processes is observed, the data are used to set the exclusion limit.

\section{Upper Limits for Instanton-induced Processes}

The upper limit is determined with a $\mathrm{CL}_{s}$ statistical analysis [11] using the the method of fractional event counting, optimised for the presence of systematic uncertainties [12], which are treated as Gaussian distributions. Statistical fluctuations are simulated using Poisson statistics.

The limit is calculated using the full range of the discriminator distribution, Fig. B. Figure $\Theta$ shows the behaviour of the observed $\mathrm{Cl}_{s}$ for different tested values of the instanton cross-section, computed with $2 \mathrm{M}$ toy $\mathrm{MC}$ simulations per point. Also the expected median upper-limit (i.e. the background-only hypothesis ) with the bands corresponding to $\pm 1 \sigma$ and $\pm 2 \sigma$ fluctuations is shown. The observed limit is $1.6 \mathrm{pb}$ at $95 \% \mathrm{CL}$ in comparison with the cross-section of $10 \mathrm{pb}$ estimated using QCDINS. 


\section{Conclusion}

A search for QCD instanton-induced processes is presented in deep-inelastic scattering at the electron-proton collider HERA in the kinematic region defined by the Bjorken-scaling variable $x>10^{-3}$, the inelasticity $0.2<y<0.7$ and the photon virtuality $150<Q^{2}<15000 \mathrm{GeV}^{2}$. The search is performed using $\mathrm{H} 1$ data corresponding to an integrated luminosity of $357 \mathrm{pb}^{-1}$.

Several observables of the hadronic final state of the events are exploited to identify a potentially instanton-enriched domain. Two Monte Carlo models, RAPGAP and DJANGOH, are used to estimate the background from the standard DIS processes, and the instanton-induced scattering processes are modeled by the program QCDINS. In order to extract the expected signal a multivariate data analysis technique is used. No evidence for QCD instanton-induced processes is observed. An upper limit of $1.6 \mathrm{pb}$ is set on the instanton cross-section at 95\% CL. This result suggests the exclusion of the theoretically predicted cross-section of $10 \mathrm{pb}$ estimated using QCDINS.

\section{Acknowledgements}

We would like to thank A. Ringwald and F. Schrempp for many helpfull discussions. We are grateful to the HERA machine engineers and technicians whose outstanding efforts have made this experiment possible. We would also thank our funding agencies and the DESY directorate for their support.

\section{References}

[1] G. 't Hooft, Symmetry Breaking Through Bell-Jackiw Anomalies, Phys. Rev. Lett. 37 (1976) 8; G. 't Hooft, Computation Of The Quantum Effects Due To A Four-Dimensional Pseudoparticle, Phys. Rev. D 14 (1976) 3432; [Erratum-ibid. D 18 (1976) 2199].

[2] A. A. Belavin, A. M. Polyakov, A. S. Shvarts and Y. S. Tyupkin, Pseudoparticle Solutions Of The Yang-Mills Equations, Phys. Lett. B 59 (1975) 85.

[3] A. Ringwald and F. Schrempp, Confronting instanton perturbation theory with QCD lattice results, Phys. Lett. B 459 (1999) 249 [hep-lat/9903039].

[4] T. Carli, J. Gerigk, A. Ringwald and F. Schrempp, QCD instanton-induced processes in deep-inelastic scattering: Search strateg, in: Monte Carlo Generators for HERA Physics (Hamburg, Germany, 1999), A. Doyle, G. Ingelman, H. Jung and G. Grindhammer, Eds, p.329, hep-ph/9906441.

[5] H. Jung, Hard diffractive scattering in high-energy e p collisions and the Monte Carlo, Comput. Phys. Commun. 86 (1995) 147;

[6] K. Charchula, G. A. Schuler and H. Spiesberger, Combined QED and QCD radiative effects in deep inelastic lepton - proton scattering: The Monte Carlo generator DJANGO6, Comput. Phys. Commun. 81 (1994) 381.

[7] A. Ringwald and F. Schrempp, QCDINS 2.0: A Monte Carlo generator for instanton-induced processes in deep-, Comput. Phys. Commun. 132 (2000) 267 [hep-ph/9911516].

[8] T. Sjostrand, "High-energy physics event generation with PYTHIA 5.7 and JETSET 7.4," Comput. Phys. Commun. 82 (1994) 74,[arXiv:hep-ph/9508391]. 
[9] G. C. Fox and S. Wolfram, "Event Shapes in e+ e- Annihilation," Nucl. Phys. B 149 (1979) 413 [Erratum-ibid. B 157 (1979) 543].

[10] A. Hocker, J. Stelzer, F. Tegenfeldt, H. Voss, K. Voss, A. Christov, S. Henrot-Versille and M. Jachowski et al., TMVA - Toolkit for Multivariate Data Analysis, PoS ACAT (2007) 040 [physics/0703039 [PHYSICS]].

[11] T. Junk, "Confidence level computation for combining searches with small statistics," Nucl. Instrum. Meth. A 434 (1999) 435 [hep-ex/9902006].

[12] P. Bock, "Computation of confidence levels for exclusion or discovery of a signal with the method of fractional event counting," JHEP 0701 (2007) 080 [hep-ex/0405072].

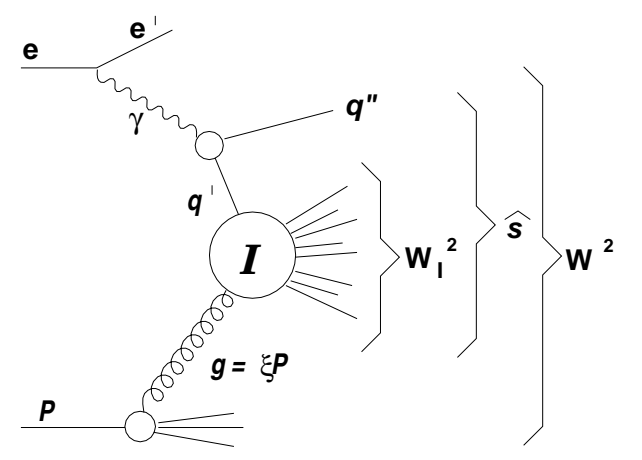

DIS variables:

$$
\begin{aligned}
& s=(e+P)^{2} \\
& Q^{2}=-\gamma^{2}=-\left(e-e^{\prime}\right)^{2} \\
& x=Q^{2} /(2 P \cdot \gamma) \\
& y=Q^{2} /(s x) \\
& W^{2}=(\gamma+P)^{2}=Q^{2}(1-x) / x \\
& \hat{s}=(\gamma+g)^{2} \\
& \xi=x\left(1+\hat{s} / Q^{2}\right)
\end{aligned}
$$

Variables of the instanton subprocess:

$$
\begin{aligned}
& Q^{\prime 2} \equiv-q^{\prime 2}=-\left(\gamma-q^{\prime \prime}\right)^{2} \\
& x^{\prime} \equiv Q^{\prime 2} /\left(2 g \cdot q^{\prime}\right) \\
& W_{I}^{2} \equiv\left(q^{\prime}+g\right)^{2}=Q^{\prime 2}\left(1-x^{\prime}\right) / x^{\prime}
\end{aligned}
$$

Figure 1: Kinematic variables of the dominant instanton induced process in DIS. Quark $q^{\prime}$, coming from virtual photon splitting, interacts with QCD instanton. Quark $q^{\prime \prime}$ becomes the outgoing quark (= current quark). 

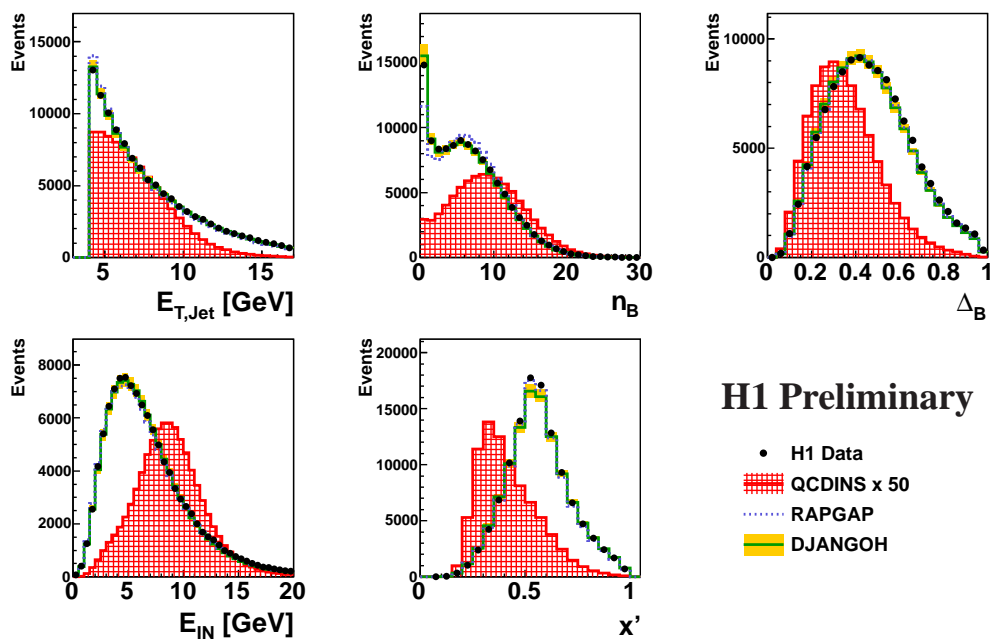

\section{H1 Preliminary}

- H1 Data

WCDINS 50

....... RAPGAP

DJANGOH

Figure 2: Distributions of the observables used in the multivariate analysis. Signal MC predictions (QCDINS) are scaled up by a factor of 50 .
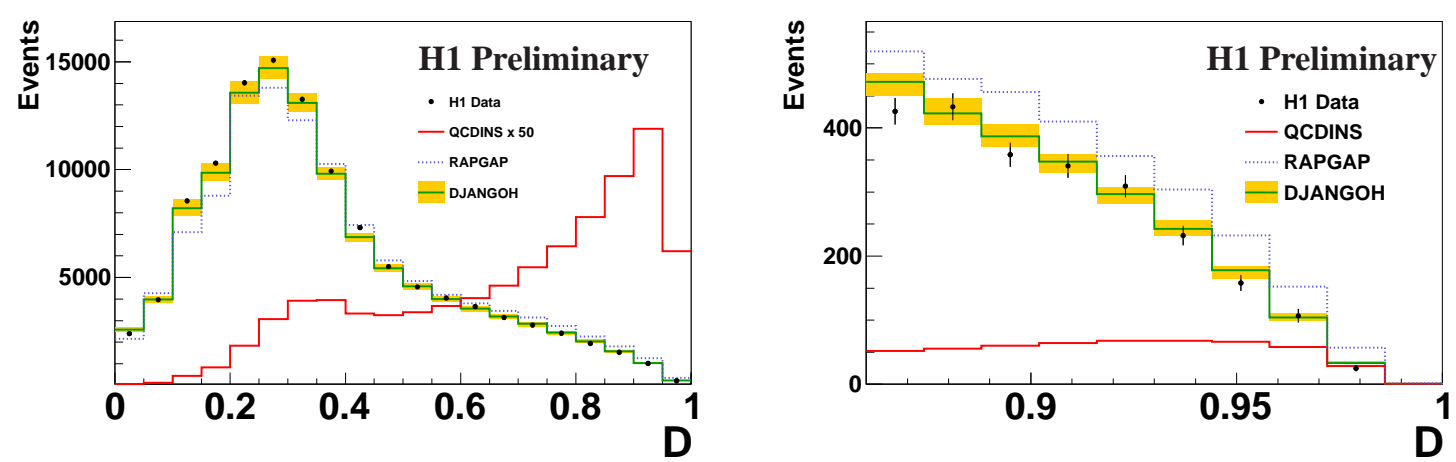

Figure 3: The distribution of the discriminator from the PDERS method in full range (left) and in the signal region, $D>0.86$ (right). Signal MC predictions (QCDINS) are scaled by a factor of 50 for the left plot. prediction are shown.

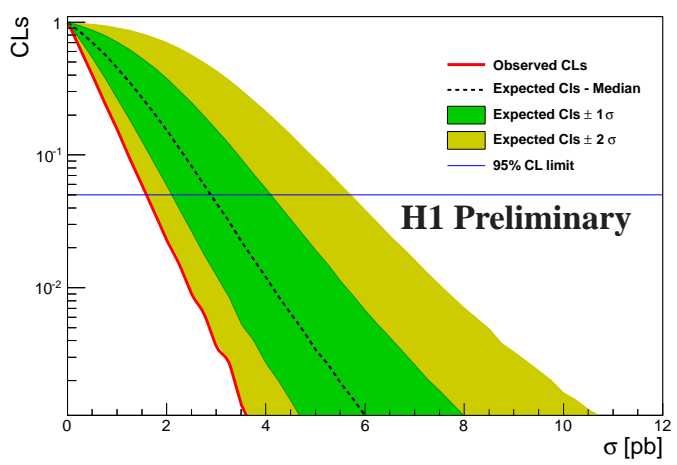

Figure 4: Observed $\mathrm{CL}_{s}$ as a function of the instanton cross section. The 95\% CL limit is indicated by the horizontal line. The dark and light bands correspond to $\pm 1 \sigma$ and $\pm 2 \sigma$ fluctuations on the expectation. 